\title{
Schematic Polymorphism in the Abella Proof Assistant
}

\author{
Gopalan Nadathur \\ University of Minnesota \\ ngopalan@umn.edu
}

\begin{abstract}
The Abella interactive theorem prover has proven to be an effective vehicle for reasoning about relational specifications. However, the system has a limitation that arises from the fact that it is based on a simply typed logic: formalizations that are identical except in the respect that they apply to different types have to be repeated at each type. We develop an approach that overcomes this limitation while preserving the logical underpinnings of the system. In this approach object constructors, formulas and other relevant logical notions are allowed to be parameterized by types, with the interpretation that they stand for the (infinite) collection of corresponding constructs that are obtained by instantiating the type parameters. The proof structures that we consider for formulas that are schematized in this fashion are limited to ones whose type instances are valid proofs in the simply typed logic. We develop schematic proof rules that ensure this property, a task that is complicated by the fact that type information influences the notion of unification that plays a key role in the logic. Our ideas, which have been implemented in an updated version of the system, accommodate schematic polymorphism both in the core logic of Abella and in the executable specification logic that it embeds.
\end{abstract}

\section{ACM Reference Format:}

Gopalan Nadathur and Yuting Wang. 2018. Schematic Polymorphism in the Abella Proof Assistant. In Proceedings of ACM Conference (Conference'17). ACM, New York, NY, USA, 12 pages. https://doi.org/10.1145/nnnnnnn.nnnnnnn

\section{INTRODUCTION}

The Abella proof assistant [1] is a vehicle for formalizing object systems that are described in a syntax-directed and rule-based fashion. Its success in this domain can be attributed to three key characteristics. First, it provides a means for constructing specifications using relations that are given via fixed-point definitions [9], an approach that works well even in the presence of non-deterministic and non-terminating behavior. Moreover, the fixed-point definitions can be specialized to yield a treatment of induction and coinduction [21]. Second, Abella facilitates a higher-order abstract syntax treatment of objects whose structures encompass bound variables. It realizes this capability by providing $\lambda$-terms as a means for representing objects, by permitting such terms to be compared modulo $\lambda$-conversion, and by incorporating a special quantifier $\nabla$ (pronounced as nabla) that allows recursive descriptions to encompass binding constructs $[1,13]$. Finally, Abella supports a two-level

Permission to make digital or hard copies of part or all of this work for personal or classroom use is granted without fee provided that copies are not made or distributed for profit or commercial advantage and that copies bear this notice and the full citation on the first page. Copyrights for third-party components of this work must be honored.

For all other uses, contact the owner/author(s).

Conference'17, fuly 2017, Washington, DC, USA

(c) 2018 Copyright held by the owner/author(s).

ACM ISBN 978-x-xxxx-xxxx-x/YY/MM.

https://doi.org/10.1145/nnnnnnn.nnnnnnn

\author{
Yuting Wang \\ Yale University \\ yuting.wang@yale.edu
}

logic approach to reasoning $[6,10]$. In this approach, object systems can be described in an expressive and executable specification logic called the logic of hereditary Harrop formulas or $\mathrm{HH}$ [12] and can subsequently be reasoned about through an embedding in the main logic underlying Abella. Since $\mathrm{HH}$ is implemented in the language $\lambda$ Prolog [12], this feature of Abella enables a transparent process of reasoning about actual programs, a capability that has, for example, been used in formalizing compiler correctness arguments [23].

The underlying basis for Abella is provided by a logic called $\mathcal{G}$ [5]. This logic is built over the expressions of the simply typed $\lambda$-calculus. As a consequence, the descriptions of objects, the definitions and the theorems that can be constructed in Abella are all monomorphically typed. This can sometimes make a formalization task more tedious and less modular than is desirable. For example, in some approaches to compiling functional programs, it is necessary to consider multiple intermediate languages, the expressions of which must be distinguished by their types. However, correctness arguments need common operations, such as combining lists of bound variables, and similar proofs of properties concerning such operations at each type. In the current system, this leads to a replication of definitions and proofs that differ only in the type to which they apply [23]. This problem carries over also to the specification logic in the two-level logic approach: the need to embed that logic within a simply typed one means that it too must be simply typed and specifications or implementations of "library" operations, such as those over lists, have to be repeated at different types.

In this paper, we develop an approach to overcoming this difficulty while retaining the logical basis of Abella. The core of our idea is simple: we parameterize specifications, definitions, theorems and proofs by types such that we can obtain the simply typed versions we want essentially by instantiating the types. However, the actual realization of this idea is more subtle than might initially be apparent. One issue arises from the need to embed schematized specifications developed in $\mathrm{HH}$ in the main logic: to support this possibility, it becomes necessary to permit the schematization of parts of a fixed-point definition in addition to allowing for the parameterization of the entire definition by types. Another issue concerns the structure of proofs. A schematic "theorem" can be part of a library only if its validity is independent of the particular type vocabulary in existence at the time that it is proved. One way to enforce this requirement is to limit attention to only those proofs for the theorem that are independent of the way in which the types that parameterize the theorem are instantiated. However, ensuring that this property holds is tricky because of a basic characteristic of $\mathcal{G}$ : type information significantly influences unification over simply typed $\lambda$-terms, an operation that is fundamental to supporting case-analysis style reasoning over fixed-point definitions.

The rest of this paper is devoted to highlighting the issues discussed above and to presenting the technical machinery that we 
have developed towards solving them. In the next section we introduce Abella and the logic underlying it. In this presentation, we simplify the logic in a way that allows us to focus on the key aspects of our work without a loss of generality. Section 3 then describes a parameterization of the core language with types; of particular interest here is the modified form for fixed-point definitions and the interpretation of parameterized theorems. Section 4 presents a lifting of the proof rules for the core logic in a way that ensures that the schematic proofs that result from using the lifted proof rules have the properties we desire of them. Section 5 illustrates the developments in the previous two sections by showing how they can be used to construct schematic proofs for some sample schematic theorems. Section 6 makes the argument that the technical developments in this paper apply to the full Abella system even though we have exposed them in a simplified setting. It does this by describing the aspects of the underlying logic that were left out earlier and by outlining how the previously presented ideas can be extended to cover them. ${ }^{1}$ We conclude the paper in Section 7 with a discussion of related work and an assessment of the enhancements to Abella that are made possible by this work. We have in fact implemented our ideas in an enhanced version of Abella that supports schematic polymorphism. Information on how to download this system can be found at the website at http://sparrow.cs.umn.edu/schmpoly. This website also contains some examples that illustrate the use of the new features of the system.

\section{THE ABELLA PROOF SYSTEM}

For most of this paper, we will limit our view of the logic underlying Abella to one that does not include co-induction and the $\nabla$ quantifier. We will also restrict the embedded specification logic to that of Horn clauses, a sublogic of HH. Our presentation of Abella in this section implicitly builds in these simplifications. We have chosen to limit our discussion in this way because it eases the presentation of the core technical ideas in our work without obscuring the issues that are essential to realizing schematic polymorphism in the full system. To validate the latter observation, we include in Section 6 a discussion of how the developments described in the earlier parts of the paper are impacted by the additional features of Abella.

\subsection{A Logic with Fixed-Point Definitions}

The logic $\mathcal{G}$ is based on an intuitionistic and predicative subset of Church's Simple Theory of Types [2]. One component of the type vocabulary is a collection of sorts that includes prop, the type for propositions, and at least one other member. In addition, the vocabulary may include type constructors, i.e. it may contain symbols such as $c$ with a designated arity $n$ that can be used with types $\alpha_{1}, \ldots, \alpha_{n}$ to yield the type $c \alpha_{1} \cdots \alpha_{n}$. The sorts and the types that are constructed in this way constitute the atomic types. The function types are constructed using the infix and right associative operator $\rightarrow$. Each well-formed type may obviously be written in the form $\alpha_{1} \rightarrow \cdots \rightarrow \alpha_{n} \rightarrow \beta$ where $\beta$ is an atomic type. When a type is rendered in this form, we refer to $\alpha_{1}, \ldots, \alpha_{n}$ as its argument types and to $\beta$ as its target type. Note that the sequence of argument types may sometimes be empty, i.e. the type may simply be of the

\footnotetext{
${ }^{1}$ This discussion also makes the case that the addition of schematic polymorphism in fact rationalizes an aspect of the Abella system that previously had an ad hoc treatment.
}

form $\beta$. A predicate type is a type with at least one argument type and a target type that is prop.

Terms are constructed from collections of typed constants and variables using abstraction and application, the latter being a left associative operator. The formation rules for terms are constrained by types and they also associate types with well-formed expressions in the usual way. Two terms are considered equal and hence interchangeable if one can be $\lambda$-converted to the other. In light of this fact, and also the fact that such a form exists for every term, we will assume that any term that we examine is in $\lambda$-normal form. The constants are sub-divided into non-logical and logical ones. Nonlogical constants are restricted in that prop must not appear in their argument types. Such a constant is called a predicate if it has a predicate type. The logical constants comprise $T$ and $\perp$ of type prop, $\wedge$, $\vee$, and $\supset$ of type prop $\rightarrow$ prop $\rightarrow$ prop, and, for each type $\alpha$ that does not contain prop, $\forall_{\alpha}$ and $\exists_{\alpha}$ of type ( $\alpha \rightarrow$ prop) $\rightarrow$ prop. Well-formed terms of the type prop are also called formulas. Formulas (in normal form) have the structure $\left(p t_{1} \cdots t_{n}\right)$ where $p$ is a constant. If $p$ is a non-logical constant, the formula is said to be atomic and it has $p$ as its head. The constants $\wedge, \vee$ and $\supset$, which correspond to the familiar propositional connectives, are written in infix form and obey the usual precedence and associativity conventions. The constants $\forall_{\alpha}$ and $\exists_{\alpha}$ constitute generalized quantifiers: the formulas $\forall_{\alpha}(\lambda x . B)$ and $\exists_{\alpha}(\lambda x . B)$ are also written as $\forall x: \alpha . B$ and $\exists x: \alpha . B$ that correspond to the more common form for quantified expressions. The formula $Q x_{1}: \alpha_{1} \ldots Q x_{n}: \alpha_{n} . F$ in which $Q$ is $\forall$ or $\exists$ will be abbreviated by $Q x_{1}: \alpha_{1}, \ldots, x_{n}: \alpha_{n}$.F. Another widely applied convention is to write a sequence of the form $x_{1}: \alpha_{1}, \ldots, x_{n}: \alpha_{n}$ in which $\alpha_{1}, \ldots, \alpha_{n}$ are identical as $x_{1}, \ldots, x_{n}: \alpha_{1}$.

Derivability in $\mathcal{G}$ is elaborated by means of a sequent calculus. The actual formulation makes explicit the eigenvariables that are used in the treatment of universal quantification. Specifically, sequents take the form $\Sigma: \Gamma \longrightarrow F$ where $\Gamma$ is a multiset of formulas, $F$ is a formula and $\Sigma$ is a collection of (typed) eigenvariables that might appear in addition to the constants in the terms in $\Gamma$ and $F$. Further, the quantifier rules are the following:

$$
\begin{aligned}
& \frac{\Sigma: \Gamma, B[t / x] \longrightarrow F}{\Sigma: \Gamma, \forall x: \alpha \cdot B \longrightarrow F} \forall \mathcal{L} \quad \frac{\sum, x: \alpha: \Gamma \longrightarrow B}{\sum: \Gamma \longrightarrow \forall x: \alpha \cdot B} \forall \mathcal{R}(x \notin \Sigma) \\
& \frac{\Sigma, x: \alpha: \Gamma, B \longrightarrow F}{\sum: \Gamma, \exists x: \alpha \cdot B \longrightarrow F} \exists \mathcal{L}(x \notin \Sigma) \quad \frac{\Sigma: \Gamma \longrightarrow B[t / x]}{\sum: \Gamma \longrightarrow \exists x: \alpha \cdot B} \exists \mathcal{R}
\end{aligned}
$$

Note that the proviso on $x$ in the $\forall \mathcal{R}$ and $\exists \mathcal{L}$ rules can always be met via $\alpha$-conversion. In the $\forall \mathcal{L}$ and $\exists \mathcal{R}$ rules, $t$ must be a term of type $\alpha$ that may use the symbols in $\Sigma$ in addition to the a priori available constants and $B[t / x]$ represents the substitution of $t$ for $x$ in $B$; we assume here and below that all substitutions are performed in a capture avoiding way. The calculus also includes rules for the other (propositional) logical constants and the usual structural rules for intuitionistic logic that we assume the reader to be familiar with.

The logic $\mathcal{G}$ actually represents a family of logics in the sense that it is parameterized by definitions. A definition is a collection of clauses that each have the form $\forall x_{1}: \alpha_{1} \ldots x_{n}: \alpha_{n} . A \triangleq B$, where $A$ is an atomic formula all of whose free variables appear in $x_{1}, \ldots, x_{n}$ and have the respective types $\alpha_{1}, \ldots, \alpha_{n}$, and $B$ is a formula all of whose free variables appear in $A$. Such a clause is 
for the predicate that is the head of $A, x_{1}: \alpha_{1}, \ldots, x_{n}: \alpha_{n}$ is called its binder and $A$ and $B$ respectively constitute its head and body. Definitions must be constructed in blocks, with all the clauses for a particular predicate confined to one block. Moreover, there is a stratification restriction on definitions: the body of a clause in a block may contain a predicate constant only if it is defined in a previous block or in the current block and, further, a predicate that is defined in the current block may not appear in the antecedent of an implication in the body. Variables in the binder of a clause can be renamed in the usual way. A definition $\mathcal{D}^{\prime}$ is a variant of another definition $\mathcal{D}$ if the clauses in $\mathcal{D}^{\prime}$ and $\mathcal{D}$ are identical but for such a renaming. A definition is named away from a set of (eigen)variables $\Sigma$ if the variables in the binders of its clauses are distinct from those in $\Sigma$.

Let us assume that the definition parameterizing $\mathcal{G}$ has been fixed to be $\mathcal{D}$. In this context, an atomic formula is considered to hold exactly when it is the head of an instance of a clause in $\mathcal{D}$ whose body also holds. This interpretation is realized through rules for introducing atomic formulas on the left and right sides of a sequent. In presenting these rules, we make use of the common view of a substitution as a mapping on variables that is then extended to a mapping on terms and we write $t \theta$ to denote the application of a substitution $\theta$ to a term $t$. The rule for introducing an atomic formula on the right, "conclusion" side of a sequent is then the following:

$$
\begin{gathered}
\frac{\sum: \Gamma \longrightarrow B \theta}{\sum: \Gamma \longrightarrow A} \text { defR } \\
\forall x_{1}: \alpha_{1} \ldots x_{n}: \alpha_{n} \cdot A^{\prime} \triangleq B \in \mathcal{D} \text { and } A^{\prime} \theta=A
\end{gathered}
$$

As should be readily apparent, this rule encodes the idea of backchaining on a clause to complete a derivation.

The rule for introducing an atomic formula on the left side of a sequent codifies a case analysis style of reasoning: to derive a sequent that has an atomic formula as an assumption, we consider the different ways in which the definition might cause an instance of the formula to hold and show that the corresponding refinements of the sequent are derivable. The following definition makes precise the cases that must be considered towards this end. Given two substitutions $\theta_{1}$ and $\theta_{2}$, we use the notation $\theta_{1} \circ \theta_{2}$ here to denote the substitution that produces the term $\left(t \theta_{2}\right) \theta_{1}$ when applied to the term $t$. Further, we write $\Gamma \theta$ to represent the result of applying the substitution $\theta$ to each element of sequence of formulas $\Gamma$. Finally, if $\Sigma$ is a collection of variables, we write $\Sigma \theta$ to denote the result of removing from $\Sigma$ the variables in the domain of $\theta$ and adding the variables that appear in the range of $\theta$.

Definition 2.1. A complete set of unifiers for two terms $t_{1}$ and $t_{2}$ of identical type is a collection $\Theta$ of substitutions such that (a) for each $\theta \in \Theta$ it is the case that $t_{1} \theta=t_{2} \theta$, and (b) for any substitution $\sigma$ such that $t_{1} \sigma=t_{2} \sigma$, there is a $\theta \in \Theta$ and a substitution $\rho$ such that $\sigma=\rho \circ \theta$. We write $\operatorname{CSU}\left(t_{1}, t_{2}\right)$ to (ambiguously) denote such a collection $\Theta$. Then, given a sequent $\mathcal{S}$ of the form $\Sigma: \Gamma, A \longrightarrow F$ where $A$ is an atomic formula, and a definition $\mathcal{D}, \operatorname{cases}(\mathcal{S}, \mathcal{D})$ (ambiguously) denotes the following set of sequents:

$$
\begin{aligned}
& \{\Sigma \theta: \Gamma \theta, B \theta \longrightarrow F \theta \mid \\
& \left.\quad \forall x_{1}: \alpha_{1} \ldots x_{n}: \alpha_{n} \cdot A^{\prime} \triangleq B \in \mathcal{D} \text { and } \theta \in \operatorname{CSU}\left(A, A^{\prime}\right)\right\}
\end{aligned}
$$

The desired inference rule can be formalized using the above definition as follows:

$$
\frac{\operatorname{cases}\left(\Sigma: \Gamma, A \longrightarrow F, \mathcal{D}^{\prime}\right)}{\Sigma: \Gamma, A \longrightarrow F} \operatorname{def\mathcal {L}}
$$

$\mathcal{D}^{\prime}$ is a variant of $\mathcal{D}$ named away from $\Sigma$

Observe that this rule may lead to a multiplicity of cases being considered for two different reasons: the head of a clause in $\mathcal{D}$ may unify with the atomic formula in the conclusion of the rule in more than one way and more than one clause in the definition may unify with this atom.

When the clauses for a particular predicate in definition $\mathcal{D}$ are finite in number, then this ensemble may also be designated as inductive. The following induction rule may be used in derivations if the clauses for $p$ are inductive:

$$
\begin{gathered}
\left\{\overline{x: \alpha}: B[S / p] \underset{S}{\longrightarrow} \overline{t^{\prime}} \mid \forall \overline{x: \alpha} \cdot p \overline{t^{\prime}} \triangleq B \in \mathcal{D}\right\} \\
\Sigma: \Gamma, S \bar{t} \longrightarrow F \\
\Sigma: \Gamma, p \bar{t} \longrightarrow F
\end{gathered}
$$

The notation - used here represents sequences (or sets shown as sequences) of expressions whose shape is indicated by $\cdot$. The term that instantiates $S$ in a use of this rule is referred to as the inductive invariant. This term must contain no free variables and its type must be the same as that of $p$. The rule formalizes the intuition that if $p$ is defined inductively and $S$ satisfies all the clauses for $p$ then $S \bar{t}$ must hold whenever $p \bar{t}$ does; hence $F$ must follow from $\Gamma$ and $p \bar{t}$ if it follows from $\Gamma$ and $S \bar{t}$.

\subsection{Constructing Proofs in Abella}

Abella is a vehicle for interactively identifying types, constants and definitions in $\mathcal{G}$ and then trying to prove relevant assertions. Showing that a formula $F$ holds amounts to constructing a proof for the sequent $\cdot: \cdot \longrightarrow F$. Abella supports a tactics-style approach based on the inference rules of $\mathcal{G}$ towards this end. We illustrate the structure of this system through a few simple reasoning examples. In this discussion we focus mainly on the treatment of the definition rules, assuming that the reader would be familiar with the treatment of the remaining rules from other contexts.

The examples that we consider concern representing and reasoning about relations on lists. We assume that the type vocabulary includes the two sorts $\iota$ and list in addition to prop. We further assume that the availability of the nonlogical constants nil and $::$ of types list and $\iota \rightarrow$ list $\rightarrow$ list respectively that provide us a means for constructing the commonly used representations of lists in $\mathcal{G}$. To simplify notation, we will write : : as an infix and right associative operator. In this context let us consider a definition with the following inductive clauses for the predicate app of type list $\rightarrow$ list $\rightarrow$ list $\rightarrow$ prop:

$$
\begin{aligned}
& \forall l: l \text { ist.app nil } l l \triangleq \top \\
& \forall x: l, l_{1}: \text { list, } l_{2}: l \text { ist, } l_{3}: \text { list. } \\
& \quad \operatorname{app}\left(x:: l_{1}\right) l_{2}\left(x:: l_{3}\right) \triangleq \text { app } l_{1} l_{2} l_{3}
\end{aligned}
$$

As is perhaps evident, these clauses identify app to be the append relation on lists. Once we have them, we can pose the usual logic programming style queries about app. An example of such a query would be the question of whether the formula $\exists l$ : list.app nil $l l$ holds. A proof of the corresponding sequent can be constructed by 
using the first clause for app in a backchaining mode via the defR rule to derive the sequent obtained by instantiating the quantifier in the formula with nil.

The fact that definitions have a fixed-point nature that goes beyond the logic programming interpretation of clauses becomes clear when we consider formulas in which atomic app formulas appear negatively. For example, consider proving

$$
\forall l_{1}: \text { ist, } l_{2} \text { : list.app nil } l_{1} l_{2} \supset l_{1}=l_{2} \text {; }
$$

the equality relation used here represents $\lambda$-convertibility. We can reduce this task through a few obvious steps to deriving the sequent

$$
\left(l_{1}: \text { list, } l_{2}: \text { list }\right) \text { : app nil } l_{1} l_{2} \longrightarrow l_{1}=l_{2} \text {. }
$$

Using the $\operatorname{def} \mathcal{L}$ rule will lead us to consider the values for $l_{1}$ and $l_{2}$ under the different ways in which app nil $l_{1} l_{2}$ can hold. The head for only the first clause for app unifies with this formula and the proof can be concluded by noting that in this case $l_{1}$ and $l_{2}$ must be identical.

Many interesting properties need inductive arguments. An example of such a property is the assertion that app is functional in its first two arguments:

$$
\begin{aligned}
& \forall l_{1}: \text { list, } l_{2}: \text { list, } l_{3}: \text { list, } l_{4}: \text { list. } \\
& \quad \text { app } l_{1} l_{2} l_{3} \supset \text { app } l_{1} l_{2} l_{4} \supset l_{3}=l_{4} .
\end{aligned}
$$

While the $\mathcal{I L}$ rule is the basis for inductive arguments, using it directly usually leads to a complex construction. Abella provides a mechanism that is grounded in the $\mathcal{I L}$ rule but that yields more intuitive proofs. This mechanism can be used to establish a formula of the form $\forall \overline{x: \alpha} \cdot F_{1} \supset \cdots \supset F_{n} \supset C$. Specifically, it can be invoked with respect to some $F_{i}$ in this formula if $F_{i}$ is atomic and it has as its head a predicate whose clauses are marked as inductive. The result of doing so will be to add the formula to be proved as an induction hypothesis to the assumption set with the following proviso: it can be used only by matching $F_{i}$ with an assumption formula that is obtained by "unfolding" the version of $F_{i}$ in the formula to be proved using a definition clause. The proviso is realized by marking the predicate head of $F_{i}$ in the unfolded form with * and in the original version with @. To illustrate the process, let us assume that $F$ represents the formula

$$
\begin{aligned}
& \forall l_{1}: \text { list, } l_{2}: \text { list, } l_{3}: \text { ist, } l_{4}: \text { list. } \\
& \qquad \text { app* }_{1} l_{2} l_{3} \supset \text { app } l_{1} l_{2} l_{4} \supset l_{3}=l_{4}
\end{aligned}
$$

Then, using the induction tactic and the rules for the logical symbols appearing in the formula, we can reduce the task of showing the functional property of app to proving the following sequent:

$$
\begin{aligned}
& \left(l_{1}: \text { list, } l_{2}: \text { list, } l_{3}: \text { list, } l_{4}: \text { ist }\right): \\
& F, \text { app }^{@} l_{1} l_{2} l_{3} \text {, app } l_{1} l_{2} l_{4} \longrightarrow l_{3}=l_{4} .
\end{aligned}
$$

At this stage, we can use the $\operatorname{def} \mathcal{L}$ rule with respect to the second assumption formula in the sequent. The case when this formula is unfolded using the first clause for app has an easy proof. Unfolding it using the second clause yields the sequent

$$
\begin{aligned}
& \left(x: \iota, l_{1}^{\prime}, l_{2}, l_{3}^{\prime}, l_{4}: \text { list }\right): \\
& \quad F \text {, app* } l_{1}^{\prime} l_{2} l_{3}^{\prime} \text {, app }\left(x:: l_{1}^{\prime}\right) l_{2} l_{4} \longrightarrow x:: l_{3}^{\prime}=l_{4} ;
\end{aligned}
$$

note the changed annotation on app that records the effect of unfolding. This sequent can be proved by using the $\operatorname{def} \mathcal{L}$ rule on the third assumption formula and then "applying" $F$ to the remaining assumption formulas.

\subsection{Embedding a Separate Specification Logic}

Relational specifications can be encoded and reasoned about directly using definitions in $\mathcal{G}$. However, Abella also allows specifications to be written in an independent, executable specification logic and then to be reasoned about through an encoding of that logic in $\mathcal{G}$. We sketch the way in which this approach is realized below.

The specification logic is based, once again, on the simply typed $\lambda$-calculus. The expressions in this logic are required to be simply typed because we desire eventually to embed them within $\mathcal{G}$. The use of the (simply typed) $\lambda$-calculus, on the other hand, is motivated by the benefits this choice provides in encoding formal systems [12]. Types and terms in the logic are constructed as in $\mathcal{G}$ with the difference that o is used for the type of propositions and the logical constants are limited to true of type $\circ, \&$ and $\Rightarrow$ of type $\circ \rightarrow 0 \rightarrow 0$ and, for each (specification logic) type $\alpha$ not containing $0, \Pi_{\alpha}$ of type $(\alpha \rightarrow 0) \rightarrow 0$. The constants \& and $\Rightarrow$ are the specification logic versions of conjunction and implication and are written in infix form with the usual precedence and associativity conventions. The family of constants $\Pi_{\alpha}$ represent universal quantification. We will write $\Pi_{\alpha}(\lambda x . F)$ as $\Pi x: \alpha . F$ and will abbreviate $\Pi x_{1}: \alpha_{1} \ldots \Pi x_{n} . \alpha_{n} F$ as $\Pi x_{1}: \alpha_{1}, \ldots, x_{n}: \alpha_{n} . F$. A term of type $\mathrm{o}$ is called a (specification logic) formula. A goal formula is a formula that is atomic, true or a conjunction of goal formulas. Relations are specified through a collection of definite clauses that are closed formulas of the form $\Pi \overline{x: \alpha} . G \Rightarrow A$ where $G$ is a goal formula and $A$ is an atomic formula. The specification logic is oriented towards constructing derivations for a closed goal formula $G$ from a specification $\Gamma$ which is a collection of definite clauses, an objective that is represented by the (specification logic) sequent $\Gamma \vdash G$. The following inference rules may be used in realizing such an objective:

$$
\begin{array}{cc}
\frac{\Gamma \vdash G_{1}}{\Gamma \vdash \text { true }} \text { true } R & \frac{\Gamma G_{2}}{\Gamma \vdash G_{1} \& G_{2}} \& R \\
\frac{\Gamma \vdash G \theta}{\Gamma \vdash A} \text { backchain } A \text { is an atomic formula } \\
\Pi \overline{x: \alpha} \cdot G \Rightarrow A^{\prime} \in \Gamma, \theta \text { is a closed substitution for } \bar{x} \text {, and } A^{\prime} \theta=A
\end{array}
$$

These rules can be attributed an execution semantics and, as such, they are part of the mechanism for interpreting specifications as programs in $\lambda$ Prolog [12].

To encode the specification logic in $\mathcal{G}$, we need first to represent its expressions. This is accomplished by lifting its type and term vocabulary into $\mathcal{G}$. The specific collection of sorts, type constructors and non-logical constants depends, of course, on the object system that we are interested in formalizing. The example that we consider below will again concern relations on lists but will represent them this time in the specification logic. In this context, it will assume the sorts $\iota$ and list. We represent atomic formulas in a way that makes them syntactically distinguishable: an atomic formula $A$ is represented by atm $A$ where atm is a constant of type $0 \rightarrow 0$.

The derivation rules of the specification logic are encoded in $\mathcal{G}$ as clauses that define a derivability predicate. To support induction on the heights of derivations, this predicate is indexed by natural numbers, which are represented by terms of type nat constructed 
using the constants $z$ and $s$ of type nat and nat $\rightarrow$ nat respectively. In the context of $\mathcal{G}$, the basis for induction is provided by the following inductive clauses for the predicate nat that has the type nat $\rightarrow$ prop:

$$
\text { nat } z \triangleq T \quad \forall n \text { : nat.nat }(\mathrm{s} n) \triangleq \text { nat } n
$$

The derivability predicate seq has the type nat $\rightarrow 0 \rightarrow$ prop and is defined by the following clauses:

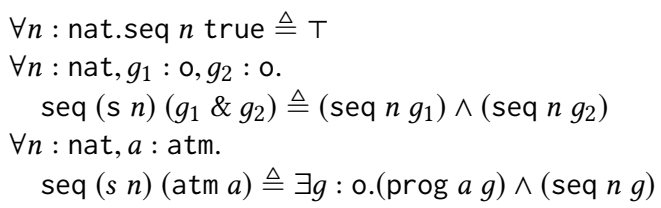

Here, the predicate prog, which has type $0 \rightarrow 0 \rightarrow$ prop, serves as the interface for encoding particular specifications that might be provided in the specification logic. For example, suppose that we have identified append of type list $\rightarrow$ list $\rightarrow$ list $\rightarrow$ o to be the specification logic encoding of the append relation on lists through the following clauses:

$$
\begin{aligned}
& \Pi l: \text { ist.true } \Rightarrow(\text { append nil } l l) \\
& \Pi x: \iota . \Pi l_{1}, l_{2}, l_{3}: \text { list. } \\
& \quad \text { append } l_{1} l_{2} l_{3} \Rightarrow \text { append }\left(x:: l_{1}\right) l_{2}\left(x:: l_{3}\right) \text {; }
\end{aligned}
$$

$\mathrm{nil}$ and : : are used here as the specification logic versions of the list constructors that are then lifted into $\mathcal{G}$. This definition translates into the following clauses for prog in $\mathcal{G}$ :

$$
\begin{aligned}
& \forall l: l \text { ist.prog }(\text { append nil } l l) \text { true } \triangleq \mathrm{T} \\
& \forall x: l, l_{1}: \text { list, } l_{2}: \text { list, } l_{3}: l \text { ist. } \\
& \quad \operatorname{prog}\left(\text { append }\left(x:: l_{1}\right) l_{2}\left(x:: l_{3}\right)\right)\left(\text { atm }\left(\text { append } l_{1} l_{2} l_{3}\right)\right) \triangleq \mathrm{T}
\end{aligned}
$$

Specifications can be constructed in a standalone mode in the specification logic and can be used as programs to realize computations. When we want to reason about such a program, we begin the process by loading the corresponding specification into Abella. Doing so lifts the vocabulary the specification introduces into $\mathcal{G}$ and adds a prog clause for each of the definite clauses it contains. At this stage, we can prove properties of the program by using the seq predicate to represent what is derivable from the program in the specification logic. A special notation is provided to make the encoding transparent: the expression $\{F\}$ represents the $\mathcal{G}$ formula $\exists n$ : nat.nat $n \wedge$ seq $n F$. Thus, the functional nature of the rendition of append in the specification logic can be expressed via the $\mathcal{G}$ formula

$$
\forall l_{1}, l_{2}, l_{3}, l_{4}: \text { list. }
$$

$\left\{\right.$ append $\left.l_{1} l_{2} l_{3}\right\} \supset$ append $\left.l_{1} l_{2} l_{4}\right\} \supset l_{3}=l_{4}$.

Applying the $\operatorname{def} \mathcal{L}$ rule to the $\{\cdot\}$ predicate translates transparently into case analysis over the specification logic clauses. For example, using it on the formula $\left\{\right.$ append $l_{1} l_{2} l_{3}$ \} will yield the two cases corresponding to \{append nil $l l\}$ (setting $l_{2}$ and $l_{3}$ to $l$ ) and \{append $\left.\left(x:: l_{1}^{\prime}\right) l_{2}\left(x:: l_{3}^{\prime}\right)\right\}$ (setting $l_{1}$ to $x:: l_{1}^{\prime}$ and $l_{3}$ to $x:: l_{3}^{\prime}$ ). The induction tactic can also be invoked with respect to the $\{\cdot\}$ predicate. Such an application amounts eventually to an induction on the height of a derivation measured by the first argument to seq It is an easy exercise to construct a proof of the functional property of append using these observations.

\section{A PARAMETERIZATION BY TYPES}

In the examples pertaining to lists that we considered in the previous section, we fixed the type of list elements to be $\iota$. Having to fix the type in this fashion is a consequence of using a simply typed language. In an actual application, we may need to consider relations both in the specification logic and in $\mathcal{G}$ over lists with different types of elements. The formalization of these relations would have a similar structure as also would the proofs of properties pertaining to them. However, the Abella system in its current form requires us to repeat the formalization and the proofs for each type at which they are needed. Our goal in this paper is to provide a means for avoiding this kind of redundancy. We aim to do this by enabling the process to be parameterized by types in a way that ensures that instantiating the parameters with actual types will yield constructions that are legitimate in the underlying (simply typed) logic. We begin the task of realizing our goal by describing in this section a modification to the language and the structure of definitions that provide the basis for the desired parameterization.

The first step in this direction is to allow variables to appear as atomic types in type expressions. We call type expressions not containing type variables concrete types or ground types. A type schema is an expression of the form $\left(\left[A_{1}, \ldots, A_{n}\right] \tau\right)$ where $A_{1}, \ldots, A_{n}$ is a sequence of distinct type variables and $\tau$ is a type expression all of whose type variables appears in $A_{1}, \ldots, A_{n}$. A term constant now has a type schema associated with it. We indicate such an association by writing $\left(c:\left[A_{1}, \ldots, A_{n}\right] \tau\right)$. For example, a parameterized development pertaining to lists would use the constants (nil : $[A] l$ ist $A)$ and $(:::[A] A \rightarrow$ list $A \rightarrow$ list $A)$, where list is now a unary type constructor. ${ }^{2}$ An instance of a constant of this kind is obtained by substituting the types $\tau_{1}, \ldots, \tau_{n}$ for $A_{1}, \ldots, A_{n}$ and its type is the corresponding instance of $\tau$. We denote such an instance by $c_{\left[\tau_{1}, \ldots, \tau_{n}\right]}$. Thus, $\mathrm{nil}_{[\iota]}$ represents an instance of nil that has the type (list $\iota$ ). In what follows, we may drop the subscript in depicting the instance of a constant if the information it provides is not important to the discussion.

Terms are formed in the same way as in $\mathcal{G}$, except that we now use instances of constants and type variables may appear in the types of term variables. A noteworthy aspect in this context is that the schematization of types allows us to view the quantifier symbols differently from before. Instead of their being distinct constants at each relevant type, we view them as the "schematic" constants $(\forall:[A](A \rightarrow$ prop $) \rightarrow$ prop $)$ and $(\exists:[A](A \rightarrow$ prop $) \rightarrow$ prop $)$ from which desired instances can be generated. Observe that the types appearing in a term may now contain type variables. A wellformed type- or term-level expression is said to be well-formed relative to a set of type variables $\Psi$ if the type variables appearing in the expression are contained in $\Psi$. We extend the notion of substitutions to also include mappings on types. We write $e[\phi]$ to denote the application of a type-level substitution $\phi$ to a type-level or term-level expression $e$ and $\phi_{1} \bullet \phi_{2}$ to denote the substitution that produces $e\left[\phi_{2}\right]\left[\phi_{1}\right]$ when applied to any term or type $e$. A term in the extended language represents the collection of terms in $\mathcal{G}$ that are obtained by substituting ground types for its type variables.

We now consider parameterizing definitions by types. Such a parameterization must occur at least at the level of definition blocks.

\footnotetext{
${ }^{2}$ This example also shows the usefulness of type constructors in the parameterization.
} 
For example, consider the definition of app from the previous section. In the schematic version, this definition should be for an app with the type $($ list $A) \rightarrow($ list $A) \rightarrow($ list $A) \rightarrow$ prop. Note also that we would want the schematization to work in such a way that each type instance that we generate of the definition block for app to be independent of all other instances of the same block. A less obvious but still important observation is that we will need type parameterization to work also at the clause level, i.e., we will want a concise, schematic way to signal the inclusion of all the type instances of a clause within a single definition block. This capability is needed to accommodate schematic polymorphism in the specification logic. To understand this, consider the definite clauses in the specification logic that we saw for the predicate append. To make these work for lists of different types, we would have to associate a type of the form $($ list $A) \rightarrow($ list $A) \rightarrow($ list $A) \rightarrow$ o with append and we would need to treat the definite clauses as if they represent the collection of all their ground type instances. This kind of an interpretation is already built at a computational level into the language $\lambda$ Prolog. To be able to reason about such a specification, we would have to embed the collection of all the instances of these definite clauses within $\mathcal{G}$. Under the embedding scheme that we have described in the previous section, this means that we would have to be able to parameterize the prog clauses that encode each of the definite clauses for append by the type of the list elements.

The above discussion motivates the following definitions.

Definition 3.1. A schematic clause is an expression of the form $[\Psi] \forall \overline{x: \alpha} . A \triangleq B$, where $\forall \overline{x: \alpha} \cdot A \triangleq B$ has the structure of a clause in $\mathcal{G}$ except that variables may appear in the types and $\Psi$ is a listing of some of the type variables appearing in the clause. All the terminology associated with clauses in $\mathcal{G}$ carries over to the schematic version. A schematic definition block comprises a finite sequence of distinct type variables $\Psi^{\prime}$, a finite set of predicate constants $\left\{c^{1}:\left[\Psi^{\prime}\right] \tau_{1}, \ldots, c^{n}:\left[\Psi^{\prime}\right] \tau_{n}\right\}$, and a collection of schematic clauses each of which is for some $c^{i}$. A schematic clause and definition block of the forms shown are said to be parameterized by $\Psi$ and $\Psi^{\prime}$ respectively.

Definition 3.2. A schematic definition block parameterized by $\Psi^{\prime}$ with associated predicate constants $\left\{c^{1}:\left[\Psi^{\prime}\right] \tau_{1}, \ldots, c^{n}:\left[\Psi^{\prime}\right] \tau_{n}\right\}$ is well-formed if, for every clause $[\Psi] \forall \overline{x: \alpha} . A \triangleq B$ in it, it is the case that (a) $\Psi$ is disjoint from $\Psi^{\prime}$, (b) $A$ and $B$ are well-formed in $\Psi \cup \Psi^{\prime}$, (c) all occurrences of $c^{i}$ in $A$ and $B$ are at the instance $c_{\left[\Psi^{\prime}\right]}^{i}$, and (d) all the type variables that occur in $B$ also occur in $A$.

The wellformedness definition above requires the constants $c^{1}, \ldots$, $c^{n}$ introduced by a schematic definition block to be used at their "defined types" at every occurrence in the block. This condition ensures the independence of each instance of such a block from every other instance. If $\mathcal{B}$ is a schematic definition block, we will write $\mathcal{B}_{[\bar{\tau}]}$ to represent an instance of the block that is obtained by substituting the type expressions $\bar{\tau}$ for the type variables that parameterize $\mathcal{B}$. Subsequent to the block, each $c^{i}$ is treated as having the type schema $\left[\Psi^{\prime}\right] \tau_{i}$ associated with it. The requirement that all the type variables in the body of a schematic clause also occur in the head has a technical motivation: it ensures that the type instance of the body is fixed as soon as the type instance of the head is determined, a property that will become important when we consider the construction of proofs.

A schematic definition block serves as an abbreviated representation of a collection of definition blocks in $\mathcal{G}$ that are obtained as follows. First, we instantiate the type variables that parameterize the block with concrete types. Within the structure thus obtained, we generate all the versions of each schematic clause by instantiating the type variables that parameterize the clause with all available concrete types. Note that both the collection of definition blocks and the collection of clauses within each block that are generated in this way are sensitive to the vocabulary of types in existence at a particular point. However, the schematic proofs whose construction we will support will be such that they will allow us to prove only those statements whose instances have derivations in $\mathcal{G}$ independently of the available type signature.

We adopt also a schematic view of the properties we would like to prove in the context of a schematic definition. A schematic formula is an expression of the form $\left[A_{1}, \ldots, A_{n}\right] F$ in which $F$ is a well-formed formula relative to the collection of type variables $A_{1}, \ldots, A_{n}$. We say that such a formula is parameterized by the type variables $A_{1}, \ldots, A_{n}$. Given the ground types $\tau_{1}, \ldots, \tau_{n}$, we can generate the formula $F\left[\tau_{1} / A_{1}, \ldots, \tau_{n} / A_{n}\right]$ in $\mathcal{G}$. A schematic formula is considered a schematic theorem only when any of its type instances generated in this way is a theorem of $\mathcal{G}$. A schematic theorem not parameterized by any type variable coincides with a theorem in $\mathcal{G}$.

\section{SCHEMATIZING PROOFS}

Schematic theorems must be such that their type instances hold in $\mathcal{G}$ regardless of the type vocabulary. While there can be different approaches to establishing such theorems, our focus here will be on what we call schematic proofs. These are structures associated with schematic formulas that yield proofs in $\mathcal{G}$ of type instances of the formulas simply by instantiating the type variables that appear in them. For us to be able to generate such a structure, it must be the case that a type instance of the schematic formula has a proof in $\mathcal{G}$ that does not use information specific to that instance. This is true of many proofs in $\mathcal{G}$. For example, the proofs we sketched in Section 2 for the different properties of app did not depend on the element type being $\iota$. Our objective in this section is to lift the proof rules for $\mathcal{G}$ to apply to schematic formulas in such a way that they yield schematic proofs. The main challenge in realizing this objective is articulating a schematic version of the $\operatorname{def} \mathcal{L}$ rule: unification plays a fundamental role in the formulation of this rule and unification over simply typed $\lambda$-terms depends significantly on type information [16].

\subsection{Schematizing sequents and the basic rules}

The lifted versions of our proof rules will apply to schematic sequents that have the form $\Psi ; \Sigma: \Gamma \longrightarrow B$. These sequents augment the ones in $\mathcal{G}$ with a set $\Psi$ of type variables that binds the type variables in $\Sigma, \Gamma$ and $B$. This set will remain unchanged throughout the derivation of the sequent. Thus, the variables in this set will function as placeholders for arbitrary types but will be like "black boxes" in that they will not allow us to look at or use the particular 
structures of the types that fill them. To prove a schematic formula $\left[A_{1}, \ldots, A_{n}\right] F$, we will need to derive the schematic sequent $A_{1}, \ldots, A_{n} ; \emptyset: \emptyset \longrightarrow F$.

The schematic versions of the rules for the logical symbols in $\mathcal{G}$ are obtained essentially by adding a set of type variables, represented by a schema variable such as $\Psi$, to the sequents that form the premises and conclusions of the rules in $\mathcal{G}$. For example, the following constitute schematic versions of the quantifier rules:

$$
\begin{gathered}
\frac{\Psi ; \Sigma: \Gamma, B[t / x] \longrightarrow F}{\Psi ; \Sigma: \Gamma, \forall x: \alpha \cdot B \longrightarrow F} s-\forall \mathcal{L} \quad \frac{\Psi ; \Sigma: \Gamma \longrightarrow B[t / x]}{\Psi ; \Sigma: \Gamma \longrightarrow \exists x: \alpha \cdot B} s-\exists \mathcal{R} \\
\frac{\Psi ; \Sigma, x: \alpha: \Gamma \longrightarrow B}{\Psi ; \Sigma: \Gamma \longrightarrow \forall x: \alpha \cdot B} s-\forall \mathcal{R}(x \notin \Sigma) \\
\frac{\Psi ; \Sigma, x: \alpha: \Gamma, B \longrightarrow F}{\Psi ; \Sigma: \Gamma, \exists x: \alpha \cdot B \longrightarrow F} s-\exists \mathcal{L}(x \notin \Sigma)
\end{gathered}
$$

As before, $t$ must be a term of type $\alpha$ in these rules and it must be constructed using only the available constants and the symbols in $\Sigma$. It is easy to see the schematic nature of these rules: by instantiating the premises and conclusions of each rule with any substitution of ground types for variables in $\Psi$, we get a rule in $\mathcal{G}$. The rules for the remaining logical symbols have a similarly obvious structure and quality.

\subsection{Schematic rules for fixed-point definitions}

The schematic forms of the rules for introducing atomic formulas in sequents have to pay more careful attention to the interpretation of type variables. The following definition will be useful in formalizing these rules.

Definition 4.1. The reduced form of a schematic clause $[\Psi] C$ that appears in a schematic definition block parameterized by $\Psi^{\prime}$ is $\left[\Psi^{\prime \prime}\right] C$ where $\Psi^{\prime \prime}$ contains type variables from $\Psi$ and $\Psi^{\prime}$ only if they occur in $C$. Note that because the type variables in the body of the clause must occur in its head, $\Psi^{\prime \prime}$ contains exactly the type variables that occur in the head of $C$. We write $\mathcal{D}_{\mathcal{R}}$ to represent a collection of the reduced forms of the schematic clauses in the definition $\mathcal{D}$.

The main issue in formalizing the schematic version of the defR rule, which we denote by $s$-defR, is that we have to consider instantiating the type variables in schematic clauses. This rule is presented below.

$$
\begin{gathered}
\frac{\Psi ; \Sigma: \Gamma \longrightarrow B \theta[\phi]}{\Psi ; \Sigma: \Gamma \longrightarrow A} s \text {-defR } \\
{\left[\Psi^{\prime}\right] \forall \overline{x: \alpha} \cdot A^{\prime} \triangleq B \in \mathcal{D}_{\mathcal{R}} \text { and } A^{\prime} \theta[\phi]=A}
\end{gathered}
$$

In this rule $\phi$ and $\theta$ are, respectively, a type substitution for $\Psi^{\prime}$ and a term substitution for $\bar{x}$ whose range is well-formed with respect to $\Psi$.

As we have already noted, the schematization of the $\operatorname{def} \mathcal{L}$ rule is complicated by the fact that the structure of unifiers for terms in the simply typed $\lambda$-calculus can depend on types. To circumvent this difficulty, we will limit ourselves to those situations in which the complete set of unifiers (CSUs) have the same structure no matter how type variables are instantiated. At a practical level, such unifiers can be computed, for instance, by using higher-order pattern unification that does not pay attention to type information $[11,17]$. Note that the described constraint on CSUs must hold independently of the extent of the type vocabulary. For this reason, we lift the notion of CSUs in Definition 2.1 to encompass unification of terms containing type variables, where type variables are treated as "frozen" typelevel constants. Since there is an injection from the concrete types that can be constructed under any possible extension to the existing type vocabulary to the types that can be constructed from the existing type vocabulary plus the infinite set of type variables, it suffices to consider CSUs that are "type-generic" in the latter context.

Definition 4.2. A type-generic complete set of unifiers for two schematic terms $t_{1}$ and $t_{2}$ of identical type that are parameterized by the type variables $A_{1}, \ldots, A_{n}$ is a collection $\Theta$ of substitutions such that $\left\{\theta\left[\tau_{1} / A_{1}, \ldots, \tau_{n} / A_{n}\right] \mid \theta \in \Theta\right\}$ is a complete set of unifiers for $t_{1}\left[\tau_{1} / A_{1}, \ldots, \tau_{n} / A_{n}\right]$ and $t_{2}\left[\tau_{1} / A_{1}, \ldots, \tau_{n} / A_{n}\right]$ for any type expressions $\tau_{1}, \ldots, \tau_{n}$. We write $\operatorname{CSU}_{\text {gen }}\left(t_{1}, t_{2}\right)$ to (ambiguously) denote such a collection $\Theta$.

We now describe conditions under which we can identify a schematic version of case analysis over an atomic formula.

Definition 4.3. Let $\mathcal{S}=(\Psi ; \Sigma: \Gamma, A \longrightarrow D)$ be a schematic sequent in which $A$ is an atomic formula and let

$$
C=\left(\left[\Psi^{\prime}\right] \forall \overline{x: \alpha} \cdot A^{\prime} \triangleq B\right)
$$

be a schematic clause named away from $\Sigma$ and such that $\Psi^{\prime}$ is disjoint from $\Psi$. Then $\mathcal{S}$ is analyzable in a generic way with respect to the clause $C$ on $A$ and the analysis produces the corresponding set cases $_{\text {gen }}(\mathcal{S}, C)$ if one of the following conditions hold:

(1) $A$ and $A^{\prime}$ are not unifiable under any instantiation of type variables. In this case, cases $_{\text {gen }}(\mathcal{S}, \mathcal{C})=\emptyset$.

(2) There is a type substitution $\phi$ for the variables in $\Psi^{\prime}$ whose range is well-formed in $\Psi$ and there is a type generic $\operatorname{CSU} \Theta$ for $A$ and $A^{\prime}[\phi]$ such that, for any type substitution $\phi^{\prime}$ that makes $A\left[\phi^{\prime}\right]$ and $A^{\prime}\left[\phi^{\prime}\right]$ unifiable, there is a type substitution $\delta$ such that $\phi^{\prime}=\delta \bullet \phi$. In this case, $\operatorname{cases}_{\text {gen }}(\mathcal{S}, \mathcal{C})$ denotes the set $\{\Psi ; \Sigma \theta: \Gamma \theta, B[\phi] \theta \longrightarrow D \theta \mid \theta \in \Theta\}$.

$\mathcal{S}$ is amenable to case analysis on A against a schematic definition $\mathcal{D}$ if it is analyzable with respect to every clause in $\mathcal{D}_{\mathcal{R}}$ on $A$ and the case analysis then results in the set of sequents

$$
\operatorname{cases}_{\text {gen }}\left(\mathcal{S}, \mathcal{D}_{\mathcal{R}}\right)=\left\{\mathcal{S}^{\prime} \mid \mathcal{C} \in \mathcal{D}_{\mathcal{R}}, \mathcal{S}^{\prime} \in \text { cases gen }_{\text {gen }}(\mathcal{S})\right\} .
$$

The conditions governing the analyzability of a schematic sequent with respect to a schematic clause can be understood as follows. If the first condition is satisfied, then we can use a generic rule with an empty set of premises deriving from the clause being considered. If the second condition is satisfied and we can find a type substitution and a type generic CSU satisfying its requirements, we can once again deal generically with this clause: the premise sequents that need to be considered in a $\operatorname{def} \mathcal{L}$ rule for a type instance of the conclusion sequent in $\mathcal{G}$ will be a type instance of the set of sequents produced by the analysis.

Using the above definition, a schematic version of the definition left rule can be formulated as follows: 


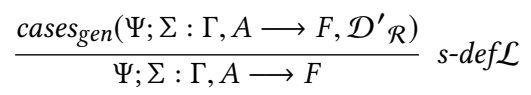$$
\mathcal{D}^{\prime} \text { is a variant of } \mathcal{D} \text { named away from } \Sigma \text { and } \Psi
$$

The rule is governed by a proviso: it can be used only if the lower sequent is amenable to case analysis on $A$ against the operative (schematic) definition $\mathcal{D}$.

In Section 5, we will illustrate how this rule can be used in constructing schematic proofs. In understanding the content of the rule, it is useful to also see situations in which it is not applicable. Suppose that we have the constant $\mathrm{p}:[A] A \rightarrow \iota$ and the clause $\forall x: A$. eq $_{[A]} x x \triangleq \mathrm{T}$ for the predicate eq $:[A] A \rightarrow A \rightarrow$ prop and we want to prove the formula

$$
[A, B] \forall(x: A)(y: B) \cdot \mathrm{eq}_{[\iota]}\left(\mathrm{p}_{[A]} x\right)\left(\mathrm{p}_{[B]} y\right) \supset \perp
$$

This task can be reduced to proving the following schematic sequent:

$$
A, B ; x: A, y: B: \mathrm{eq}_{[\iota]}\left(\mathrm{p}_{[A]} x\right)\left(\mathrm{p}_{[B]} y\right) \longrightarrow \perp
$$

We might want to apply the $s$ - $\operatorname{def} \mathcal{L}$ to the only assumption atom here but unfortunately neither of the conditions in Definition 4.3 holds. On the one hand, the atomic formula is unifiable with the head of the clause for eq in the case that $A$ and $B$ are set to the same type. On the other hand, a type generic CSU does not exist for the atomic formula and a type instance of the head of the clause for eq because the unifiability of their instances depends on whether or not the same types are substituted for $A$ and $B$.

\subsection{The schematic induction rule}

A schematic definition block is said to be accommodative to induction if every schematic clause within the block is parameterized by an empty sequence of type variables. If a block has this character, then each of its schematic clauses gives rise to exactly one clause in the definition block in $\mathcal{G}$ that is generated by instantiating the type variables parameterizing the block with concrete types. Thus, every instance of such a schematic definition block has a finite number of clauses and hence all these clauses can be designated as inductive ones. In consonance with this observation, we allow a schematic definition block that is accommodative to induction to be designated as inductive.

A schematic version of the induction rule $\mathcal{I L}$ is then given as follows:

$$
\begin{gathered}
\left\{\Psi ; \overline{x: \alpha}: B[S / p] \longrightarrow S \overline{t^{\prime}} \mid \forall \overline{x: \alpha} \cdot p_{[\bar{\tau}]} \overline{t^{\prime}} \triangleq B \in \mathcal{B}_{[\bar{\tau}]}\right\} \\
\Psi ; \Sigma: \Gamma, S \bar{t} \longrightarrow F
\end{gathered}
$$

This rule essentially parameterizes the conclusion and premises of the $\mathcal{I L}$ rule with $\Psi$. As in the case of the $\mathcal{I L}$ rule, the type of the term that instantiates $S$ must be identical to that of $p_{[\bar{\tau}]}$. Moreover, the type variables appearing in this term must be contained in $\Psi$. It is easy to see that this rule reduces to $\mathcal{I L}$ under the instantiation of $\Psi$ with ground types. Although the rule that we have presented here is applicable only to schematic definition blocks that have defining clauses for exactly one predicate, it can be generalized to deal with predicates that are defined mutually inductively. We elide a discussion of the generalized rule in this paper.

\subsection{Soundness of the schematic proof system}

The schematic nature of the lifted proof system we have presented can be articulated via a soundness theorem.

Theorem 4.4. Let $\Pi$ be a derivation of the schematic sequent

$$
\Psi ; \Sigma: \Gamma \longrightarrow B
$$

that is constructed using the schematic proof rules we have described. If $\Phi$ is a substitution that maps each variable in $\Psi$ to a ground type, then $\Pi[\Phi]$ is proof in $\mathcal{G}$ for the sequent $\Sigma[\Phi]: \Gamma[\Phi] \longrightarrow B[\Phi]$.

Proof. Only a sketch is provided here. The proof proceeds by induction on the height of $\Pi$, considering the different possibilities for the last (schematic) inference rule in the derivation. The argument follows an obvious pattern in most cases: We invoke the induction hypothesis on the premises of the last rule to determine that the type instances of the premise derivations will be derivations in $\mathcal{G}$ of the type instances of the corresponding premises. These derivations can then be extended by using a type instance of the last rule in the schematic proof to obtain a derivation in $\mathcal{G}$ of the desired type instance of the concluding sequent in $\Pi$; when the last rule is either $s-\exists \mathcal{R}$ or $s-\forall \mathcal{L}$, we will need the additional observation here that well-typedness of terms is preserved under type instantiation. It is now easy seen that the proof that has been generated in this way is itself the relevant type instance of $\Pi$.

The only case that needs further elaboration is that when the last rule is $s$-def $\mathcal{L}$. Given any schematic definitional clause, the proviso of this rule ensures that if the matching between the atomic formula being analyzed with the head of the clause fails (i.e., condition (1) of Definition 4.3 is satisfied), then it will also fail under the instantiation of type variables. The proviso also ensures that when the matching succeeds (i.e., condition (2) of Definition 4.3 is satisfied), it also succeeds under the type instantiation. Moreover, the type generic natural of the matching ensures the structure of the premise generated from the matching is preserved under the type instantiation. These observations allow us to complete the argument in this case as well using the previously described pattern.

The proof of the above theorem is constructive. Its procedural interpretation provides us a function for constructing proofs in $\mathcal{G}$ from the schematic proofs. A consequence of the theorem is that if a schematic formula $\left[A_{1}, \ldots, A_{n}\right] F$ has a schematic proof, then, for any ground types $\tau_{1}, \ldots, \tau_{n}, F\left[\tau_{1} / A_{1}, \ldots, \tau_{n} / A_{n}\right]$ is provable is $\mathcal{G}$.

\section{CONSTRUCTING SCHEMATIC PROOFS}

We have implemented an extension to Abella that supports schematic polymorphism based on the ideas described in this paper. We present some examples below that illustrate the new capabilities of this system and also highlight some of the limitations of the form of polymorphism it realizes.

We first consider a schematized version of the app predicate from Section 2.2. Associating the type schema $[A] l$ ist $A$ with nil and $[A] A \rightarrow$ list $A \rightarrow$ list $A$ with $::$, this predicate is defined by the following clauses in a block with the predicate signature app : [A]list $A \rightarrow$ list $A \rightarrow$ list $A \rightarrow$ prop: 


$$
\begin{aligned}
& \forall l: \text { list } A \cdot \operatorname{app}_{[A]} \text { nil } l l \triangleq \top \\
& \forall x: A . \forall l_{1}, l_{2}, l_{3}: \text { list } A . \\
& \quad \operatorname{app}_{[A]}\left(x:: l_{1}\right) l_{2}\left(x:: l_{3}\right) \triangleq \operatorname{app}_{[A]} l_{1} l_{2} l_{3}
\end{aligned}
$$

Since neither of the clauses is parameterized by a type, this block can be designated as inductive. We can get definition blocks in $\mathcal{G}$ by instantiating the variable $A$ in this schematic definition with a concrete type. Note that the blocks so generated are independent of each other and also finite in size. As such, the resulting "instance" definitions for app can also be treated as inductive in $\mathcal{G}$.

In the context of the schematic definition, we can write the following schematic formula that encodes the discussed functional property of app:

$$
\begin{aligned}
& {[A] \forall l_{1}, l_{2}, l_{3}, l_{4}: \text { list } A .} \\
& \quad \operatorname{app}_{[A]} l_{1} l_{2} l_{3} \supset \operatorname{app}_{[A]} l_{1} l_{2} l_{4} \supset l_{3}=l_{4} .
\end{aligned}
$$

The $s$ - IL rule provides the basis for lifting the annotated style of induction discussed in Section 2.2 to the schematic setting. Letting $F$ denote the formula

$$
\forall l_{1}, l_{2}, l_{3}, l_{4}: \text { list } A \cdot \operatorname{app}_{[A]}^{*} l_{1} l_{2} l_{3} \supset \operatorname{app}_{[A]} l_{1} l_{2} l_{4} \supset l_{3}=l_{4},
$$

using the induction tactic will allow us to reduce the task of proving the formula of interest to constructing a derivation for the schematic sequent

$$
\begin{aligned}
& A ;\left(l_{1}, l_{2}, l_{3}, l_{4}: \text { list } A\right): \\
& \quad F, \operatorname{app}_{[A]}^{@} l_{1} l_{2} l_{3}, \operatorname{app}_{[A]} l_{1} l_{2} l_{4} \longrightarrow l_{3}=l_{4} .
\end{aligned}
$$

This sequent is amenable to case analysis on $\operatorname{app}_{[A]}^{@} l_{1} l_{2} l_{3}$ against the operative definition: it is easy to see that there is a type generic CSU for the atom with the head of the reduced form of each app clause instantiated with $A$, thereby satisfying the second condition in Definition 4.3. The remainder of the proof follows the structure of the one sketched in Section 2.2. Note that instantiating the type variable $A$ with $\iota$ in this schematic proof will in fact yield the earlier proof.

The next example concerns the append relation defined in the specification logic. Definite clauses can also be schematized as described, e.g., in [16]. Encoding such definite clauses for append yields the following schematic clauses for prog:

$$
\begin{aligned}
& {[A] \forall l: \text { list } A . p r o g\left(\text { append }_{[A]} \text { nil } l l\right) \text { true } \triangleq \mathrm{T}} \\
& {[A] \forall x: A, l_{1}: \text { list } A, l_{2}: \text { list } A, l_{3}: \text { list } A .} \\
& \operatorname{prog}\left(\text { append }_{[A]}\left(x:: l_{1}\right) l_{2}\left(x:: l_{3}\right)\right) \\
& \quad\left(\operatorname{atm}\left(\operatorname{append}_{[A]} l_{1} l_{2} l_{3}\right)\right)
\end{aligned}
$$

The type schema $[A]$ list $A \rightarrow$ list $A \rightarrow$ list $A \rightarrow 0$ is associated with append here. Each type instance of a clause above yields a prog clause in $\mathcal{G}$ that encodes the corresponding type instance of an append clause in the specification logic. Combining the schematic clauses with the ones for seq seen earlier gives us a (schematic) encoding of the specification logic definition. The functional nature of the "polymorphic" definition of append in the specification logic can now be expressed by the following schematic formula:

$$
[A] \forall l_{1}, l_{2}, l_{3}, l_{4}: \text { list } A \text {. }
$$

$$
\text { append } \left._{[A]} l_{1} l_{2} l_{3}\right\} \supset\left\{\operatorname{append}_{[A]} l_{1} l_{2} l_{4}\right\} \supset l_{3}=l_{4} \text {. }
$$

A schematic proof can be constructed for this formula, from which the one discussed in Section 2.2 can be obtained as a type instance; we omit the details that should be easy to fill in given the discussions in Section 2.3.
Although many useful schematic theorems can be established using our schematic proof system, there are some that lie beyond its capabilities. Towards understanding the content of this observation, let us consider the schematic formula

$$
\begin{aligned}
& {[A, B] \forall x: A, f: A \rightarrow B . \exists y: B .} \\
& \quad\left(\operatorname{eq}_{[\iota]}\left(\mathrm{p}_{[A]} x\right)\left(\mathrm{p}_{[B]} y\right)\right) \vee\left(\left(\mathrm{eq}_{[\iota]}\left(\mathrm{p}_{[A]} x\right)\left(\mathrm{p}_{[B]} y\right)\right) \supset \perp\right)
\end{aligned}
$$

which uses the predicates eq and $\mathrm{p}$ introduced in Section 4.2. We see that every type instance of this formula must hold based on the following reasoning. When $A$ and $B$ are instantiated by the same type, we instantiate the existentially quantified variable $y$ with the same value that instantiates the universally quantified variable $x$ and then observe that the left branch of the disjunction holds. If $A$ and $B$ are instantiated with different types then corresponding to each term $t$ that is chosen for $x$ we pick the term $(f t)$ for $y$ and then observe that the right branch of the disjunction must hold. However, the structure of above argument depends on the type instance under consideration and hence it cannot be captured by a schematic proof. Moreover it can be checked that it is impossible to provide a schematic proof for this formula.

\section{COVERING THE FULL LOGIC}

In the discussions up to this point, we have considered only a subset of the specification logic $\mathrm{HH}$ and, correspondingly, of the logic $\mathcal{G}$. We did this so that we could focus on the main technical issues relating to our ideas for introducing schematic polymorphism. The features of $\mathrm{HH}$ and $\mathcal{G}$ that we have left out of the discussion are, however, central to the usefulness of Abella in its application domain: in particular, they are vital to the logical treatment of higher-order abstract syntax. It is important therefore that the techniques described for the simplified logics be extendable to the fully featured logics. This is indeed the case, as we try to demonstrate in this section. A complete development for the full logic that elaborates on the ideas discussed here can be found in Wang's doctoral thesis [22]. Our implementation of "schematic Abella" is, in fact, based on this complete development.

\subsection{Additional logical features of $\mathrm{HH}$ and $\mathcal{G}$}

The features that we have omitted in the earlier discussions are the $\nabla$ quantifier in $\mathcal{G}$ and the hypothetical and generic forms for goals in $\mathrm{HH}$. These features conspire to provide an inductive treatment of syntax even in the presence of binding constructs. We describe the richer forms to the logics and also motivate their usefulness below.

The enrichment to Horn clauses that results in $\mathrm{HH}$ is easy to describe at the syntactic level: goal formulas are allowed to contain universal quantifiers and implications of the form $D \Rightarrow G$ where $D$ is a definite clause. Note that this change impacts also the syntax of definite clauses which, in the enriched form, are referred to as hereditary Harrop formulas. Permitting universal quantifiers in goals implies that the vocabulary for constructing terms might change in the course of a derivation. This aspect is accounted for by changing the form of the specification logic sequent to $\Xi: \Gamma \vdash G$, where $\Xi$ represents an eigenvariable context. The earlier present rules for the specification logic are modified in an obvious way to take into account the richer structure for sequents. We additionally have the following rules to treat the new forms for goal formulas: 


$$
\frac{\Xi: \Gamma, D \vdash G}{\Xi: \Gamma \vdash D \Rightarrow G} \Rightarrow R \quad \frac{\Xi, x: \alpha: \Gamma \vdash G}{\Xi: \Gamma \vdash \Pi x: \alpha \cdot G} \Pi R
$$

The new forms for goals in $\mathrm{HH}$ add a dynamic character to both the signature and the program context that is relevant in a derivation. This feature turns out to be quite useful in capturing recursion over binding constructs. When carrying out an analysis over the body of such a construct, it is necessary to distinguish occurrences of the bound variable and it may also be necessary to attribute specific properties to the bound variable for the duration of the analysis. These are exactly the capabilities provided by universal quantifiers and implications in goals. We refer the reader to [12], amongst other sources, for concrete examples that use these observations in constructing specifications related to syntactic structure in which binding is an important component.

In reasoning about syntax, it is often necessary to treat entities such as bound variables as atomic, unanalyzable components. The universal quantifier provides this ability in $\mathrm{HH}$. However, this quantifier cannot be used for a similar purpose in $\mathcal{G}$ because the logic gives it an extensional reading; this interpretation is manifest, for example, in the $\operatorname{def} \mathcal{L}$ rule which considers the different ways in which eigenvariables may be instantiated towards making an assumption formula hold. The $\nabla$ quantifier in $\mathcal{G}$ can be understood as a new "generic" quantifier that fills this gap. ${ }^{3}$ Formally, we may construct a proof of a sequent in which the formula $\nabla x$. $(B x)$ occurs on the right by picking a new constant $c$ that does not appear in the sequent and then proving the sequent that results from replacing $\nabla x$. $(B x)$ with $(B c)$. The constants that are to be used in this way in proofs belong to a special category called the nominal constants. The treatment of $\nabla x$. $(B x)$ when it appears on the left side of the sequent, i.e., as an assumption formula, is symmetric: we get to use $(B c)$ where $c$ is a fresh nominal constant as an assumption instead.

In addition to their use in formulas, the $\nabla$ quantifier can also be used in the head of a clause in a definition in $\mathcal{G}$ [5]. More specifically, the full form for such clauses is the following:

$$
\forall \overline{x: \alpha} \cdot(\nabla \overline{z: \tau} \cdot A) \triangleq B .
$$

In generating instances of such a clause, the $\nabla$ quantifiers over the head may be instantiated by any collection of distinct nominal constants of suitable types. Further, the universal quantifiers over the clause may be instantiated by arbitrary terms of the requisite types with the proviso that they must not contain any of the nominal constants used to instantiate the $\nabla$ quantifiers. Thus, the order of the quantifiers facilitates the encoding of dependency information. For example, consider the (extended) clause

$$
\forall t: \iota .(\nabla x: \iota . \text { fresh } x t) \triangleq \mathrm{T} ;
$$

fresh is assumed to be a predicate of type $\iota \rightarrow \iota \rightarrow$ prop here This clause codifies the requirement that (fresh $x t$ ) holds exactly when $x$ is a nominal constant that does not appear in the term $t$. This capability has a general use in the context of reasoning about syntactic structures in the presence of binding: it allows us to make explicit properties such as the distinctness of variable occurrences that are captured by different binders and the uniqueness of assignments to such variables when these variables are represented by nominal constants.

\footnotetext{
${ }^{3}$ This quantifier has other uses, such as in encoding the uniqueness of names. We defer a more detailed discussion of these aspects to, e.g., [1]
}

\subsection{Schematization applied to the full system}

The additional features in $\mathrm{HH}$ and $\mathcal{G}$ interact in a benign way with our ideas related to parameterization based on types. In fact, parameterization provides a more systematic treatment of one aspect in comparison with the simply typed version of the system. This aspect concerns the encoding of $\mathrm{HH}$ in $\mathcal{G}$. We discuss these matters below.

Following the lines described in Section 2.3, the encoding of $\mathrm{HH}$ is realized by lifting its signature into $\mathcal{G}$ and by capturing its derivability relation in a definition. In encoding the derivability relation, we now also have to account for the changing nature of the signature and the program context. Modelling the changes to the signature is simplified by the presence of the $\nabla$ quantifier in $\mathcal{G}$ : this quantifier can be used to introduce a nominal constant that implicitly encodes the desired signature enhancement. To deal with the former aspect, we augment the seq predicate with an additional parameter that represents a list of the specification logic clauses that are added dynamically in the process of searching for a derivation.

Playing the above ideas out in detail leads to seq now having the type nat $\rightarrow$ (list o) $\rightarrow$ o $\rightarrow$ prop. ${ }^{4}$ Further, the clauses defining this predicate change to the following:

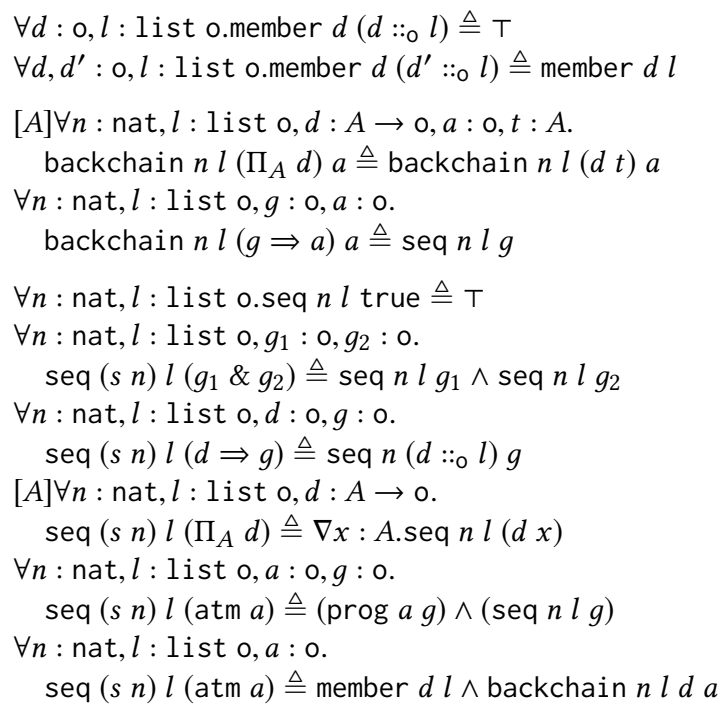

The constants backchain and member used here have the types nat $\rightarrow$ (list o) $\rightarrow$ o $\rightarrow 0 \rightarrow$ prop and o $\rightarrow$ (list o) $\rightarrow$ prop, respectively, and $\Pi$ has the schematic type $[A](A \rightarrow 0) \rightarrow 0$. The clauses for backchain encode backchaining on the definite clauses added dynamically during the derivation.

The clause for seq that treats universal goals and the clause for backchain that corresponds to instantiating a specification logic clause illuminate the usefulness of the schematic polymorphism developed in this paper in realizing a hygienic encoding of $\mathrm{HH}$. These clauses must treat quantification over variables of all possible types. The ability to parameterize the clause by the type of the variable results in a precise encoding of this fact, an improvement over the existing version of Abella in which this aspect is treated in an ad hoc way in the implementation.

\footnotetext{
${ }^{4}$ We assume that the type constructor for lists and the (schematic) list constructors have been added to the vocabulary in this discussion.
} 
The addition of $\nabla$ to the collection of logical symbols of $\mathcal{G}$ does not complicate the description of schematic proof rules for these symbols. The only remaining aspect is the schematization of the rules pertaining to the enriched form for definitions. Here we build, again, on the version of the rules described for the simply typed case. The schematic version of the (appropriate) defR and induction rules are easily obtained. To consider the $d e f \mathcal{R}$ rule as an example, in matching the atomic formula in the sequent with the head of a clause, we now have to also consider substitutions for the $\nabla$ quantified variables in the head. However, this is just as in the simply typed case and it is dealt with in a way that is orthogonal to the treatment of type variables.

There are more details to address in schematizing the $\operatorname{def} \mathcal{L}$ rule for the extended form for definitions but this can be done as we now indicate. One requirement is that we must generalize the definition of type-generic CSUs to terms containing nominal constants. The critical observation in doing this is that we can treat nominal constants whose types might contain type variables in much the same way that we have treated ordinary constants. Once we have determined this, we are able to use the original definition for type-generic CSUs (Definition 4.2) in the new context. Next, we need to generalize Definition 4.3, which describes the analysis of a sequent $\mathcal{S}$ with respect to a clause $C$, to the case where the clause has the (more general) form $\left(\left[\Psi^{\prime}\right] \forall \overline{x: \alpha} \cdot\left(\nabla \vec{z} \cdot A^{\prime}\right) \triangleq B\right)$. This is realized through two steps. First, we describe the enumeration of cases based on the possible forms of $A^{\prime}$ resulting from instantiating $\vec{z}$ with nominal constants, with the possibility that these nominal constants may be used to instantiate variables in $\mathcal{S}$. This kind of enumeration and its algorithmic treatment through unification has been considered elsewhere [5] and it is, in any case, orthogonal to the schematization of $\operatorname{def} \mathcal{L}$. Second, for each case generated in this way, we require that $S$ be analyzable in a generic way with respect to $C$ in the sense that condition (1) or (2) in Definition 4.3 is satisfied. Once Definition 4.3 has been generalized through these two steps, it can be used just as in Section 4 to describe a schematized version of the defL rule for definitions in the full form that is permitted for them in $\mathcal{G}$.

\section{RELATED WORK AND CONCLUSION}

The approach we have used in this paper to realize polymorphism by parameterizing the types of variables, constants and predicates has been influenced by previous work on functional languages (e.g., see $[3,14])$ and logic programming languages (e.g., see [7, $8,15,16])$. At a spiritual level, our development is closest to that in [16], where the parameterization is treated as a schematic one, to be understood eventually by a translation into a simply typed (logical) language. Despite the similarities in the underlying idea, we note that the context of its application and, hence, the technical challenges in making it work are significantly different. The focus in a logic programming language, for example, is on using a logic for computation, whereas the main emphasis in this paper has been to get schematic polymorphism to work in constructing proofs in a logic that includes case analysis over fixed-point definitions. Moreover, we have had the additional concern of enabling reasoning in this logic over programs in a logic programming language that itself supports schematic polymorphism.
There are other systems that support higher-order abstract syntax and that provide reasoning capabilities similar to Abella. Two specific systems that do this are Twelf [18] and Beluga [19]. These systems currently lack support for polymorphism. We believe that the ideas presented in this paper can be used to endow them as well with this feature. To understand this point, note that the only major difficulty in implementing schematic polymorphism in Twelf or Beluga lies in the formalization of case analysis in a way that is not sensitive to type information. Note also that, in order to be able to provide faithful encodings of object systems, any such analysis must be based on the structures of terms. With this understanding of the role of terms and some reflection on the intended fixed-point reading of specifications in these systems, it becomes clear that unification plays a vital role in realizing case analysis. However, unification in the context of typed $\lambda$-calculi is typically sensitive to typing information and this turns out to be the main challenge to carrying out case analysis in a schematic way when type parameterization is permitted. The key technical novelty of our work is to treat type variables as unknown entities (or "black boxes") and to use the result of a unification computation only when it can be produced without obtaining any further information about these type entities. Using this approach, it should be possible to extend case analysis to versions of these other systems that permit type parameterization. We believe that this is a direction that is worthy of further exploration because of the light-weight way in which it is able to support a useful form of polymorphism; the method piggybacks on existing mechanisms and is therefore implementable with limited additional effort.

An alternative approach to achieving polymorphism is through a module system and instantiation of modules. Rabe and Schürmann [20] developed a module system for LF which is the specification language of Twelf. The idea is to group LF declarations and definitions into signatures. To make use of a signature in another context, they apply to the signature signature morphisms which are mappings from constants in the signature to terms in the target context. Like our work, their module system preserves the logical foundation of Twelf because all the extra devices introduced to implement the module system are elaborated into the core LF. For reasoning they simply use the mechanisms provided by Twelf such as coverage checking on these elaborated definitions. However, as they have noted in their paper, signature morphisms may not preserve the validity of the checking process. Therefore, a theorem proved in a given module may not hold under an instantiation of that module. We observe that coverage checking in Twelf, similar to case analysis in Abella, makes use of unification. Therefore, we believe that the idea of schematic polymorphism that we have developed can be used to generalize coverage checking in a way that is stable under signature morphisms.

In general-purpose theorem provers such as Coq and Isabelle, reasoning principles such as case analysis and induction are derived from mechanisms for defining inductive data types (in Coq) or algebraic data types interpreted as inductively defined sets (in Isabelle/HOL). By parameterizing such definitions with type variables, we can obtain a "type-generic" way to support case analysis and induction. Because of these differences, especially because the reasoning principles are not dependent in a fundamental way on the inductive structure of terms, our work on realizing schematic 
polymorphism does not translate directly to the context of these general-purpose theorem provers. However, there has been work on exploiting the benefits of higher-order abstract syntax and the two-level logic approach within general-purpose theorem provers by encoding a specification logic that supports higher-order abstract syntax as a library and then reasoning about specifications written in this logic through the encoding. One example of such work is embodied in the Hybrid system [4]. Supporting polymorphism in this kind of a setting will eventually become important and we believe that the ideas we have developed in this paper will be relevant to these systems too at that point.

As already noted, we have implemented a version of Abella that incorporates schematic polymorphism based on the ideas developed in this paper. This version provides several benefits over the previously available version of the system. First, it allows us to write polymorphic specifications in $\mathrm{HH}$ that can be executed as programs in $\lambda$ Prolog using previously existing capabilities and that can now be reasoned about using Abella. This support for polymorphism eliminates a lot of redundancy in programming and also makes the programs or specifications and proofs of their properties more modular. Second, even without considering the specification logic, Abella itself is able to treat polymorphic definitions and theorems. Third, the extension means we are able to create standard libraries for Abella that contain definitions of generic data structures such as lists and sets and theorems describing their properties. We have only recently completed the implementation of our system and it has therefore not seen sufficient use for us to quantify the above benefits based on actual experience. However, we have used the system in one significant project that concerns the implementation and verification of a compiler for an extension of PCF that includes recursion [22, 23]. The polymorphism enhancements helped eliminate redundancies in both the implementation and the verification in the manner described. For example, using it led to a reduction of $14 \%$, measured in terms of the lines of code, in the formalization of the library components of the project in comparison with an alternative development in the earlier version of Abella that did not support polymorphism. More details about this project and comparisons can be found at the website mentioned in the introduction. Information on how to download the enhanced system can also be found at this website.

\section{ACKNOWLEDGEMENTS}

This work has been supported by the National Science Foundation grant CCF-1617771. Opinions, findings and conclusions or recommendations that are manifest in this material are those of the participants and do not necessarily reflect the views of the NSF.

\section{REFERENCES}

[1] David Baelde, Kaustuv Chaudhuri, Andrew Gacek, Dale Miller, Gopalan Nadathur, Alwen Tiu, and Yuting Wang. 2014. Abella: A System for Reasoning about Relational Specifications. Fournal of Formalized Reasoning 7, 2 (2014). https: //doi.org/10.6092/issn.1972-5787/4650

[2] Alonzo Church. 1940. A Formulation of the Simple Theory of Types. F. of Symbolic Logic 5 (1940), 56-68.

[3] Luis Damas and Robin Milner. 1982. Principal Type Schemes for Functional Programs. In Proceedings of the ACM Conference on Principles of Programming Languages. 207-212.

[4] Amy Felty and Alberto Momigliano. 2012. Hybrid: A Definitional Two-Level Approach to Reasoning with Higher-Order Abstract Syntax. F. of Automated
Reasoning 48 (2012), 43-105.

[5] Andrew Gacek, Dale Miller, and Gopalan Nadathur. 2011. Nominal abstraction. Information and Computation 209, 1 (2011), 48-73.

[6] Andrew Gacek, Dale Miller, and Gopalan Nadathur. 2012. A two-level logic approach to reasoning about computations. F. of Automated Reasoning 49, 2 (2012), 241-273. https://doi.org/10.1007/s10817-011-9218-1

[7] Michael Hanus. 1989. Polymorphic Higher-Order Programming in Prolog. In Proceedings of the 6th Int. Conf. on Logic Programming. MIT Press, 382-397.

[8] T. L. Lakshman and Uday S. Reddy. 1991. Typed Prolog: A Semantic Reconstruction of the Mycroft-O'Keefe Type System. In Logic Programming, Proceedings of the 1991 International Symposium, Vijay A. Saraswat and Kazunori Ueda (Eds.). MIT Press, San Diego, California, USA, 202-217.

[9] Raymond McDowell and Dale Miller. 2000. Cut-elimination for a logic with definitions and induction. Theoretical Computer Science 232 (2000), 91-119.

[10] Raymond McDowell and Dale Miller. 2002. Reasoning with Higher-Order Abstract Syntax in a Logical Framework. ACM Trans. on Computational Logic 3, 1 (2002), 80-136.

[11] Dale Miller. 1991. A Logic Programming Language with Lambda-Abstraction, Function Variables, and Simple Unification. F. of Logic and Computation 1, 4 (1991), 497-536.

[12] Dale Miller and Gopalan Nadathur. 2012. Programming with Higher-Order Logic. Cambridge University Press. https://doi.org/10.1017/CBO9781139021326

[13] Dale Miller and Alwen Tiu. 2005. A proof theory for generic judgments. ACM Trans. on Computational Logic 6, 4 (Oct. 2005), 749-783. http://www. lix.polytechnique.fr/Labo/Dale.Miller/papers/tocl-nabla.pdf

[14] Robin Milner. 1978. A Theory of Type Polymorphism in Programming. F. of Computer and System Sciences 17, 3 (1978), 348-375.

[15] A. Mycroft and R. A. O'Keefe. 1984. A polymorphic type system for Prolog. Artificial Intelligence 23 (1984), 295-307.

[16] Gopalan Nadathur and Frank Pfenning. 1992. The type system of a higher-order logic programming language. In Types in Logic Programming. MIT Press, 245-283.

[17] Tobias Nipkow. 1993. Functional Unification of Higher-Order Patterns. In 8th Symp. on Logic in Computer Science, M. Vardi (Ed.). IEEE, 64-74.

[18] Frank Pfenning and Carsten Schürmann. 1999. System Description: Twelf - A Meta-Logical Framework for Deductive Systems. In 16th Conf. on Automated Deduction (CADE) (LNAI), H. Ganzinger (Ed.). Springer, Trento, 202-206.

[19] Brigitte Pientka and Joshua Dunfield. 2010. Beluga: A Framework for Programming and Reasoning with Deductive Systems (System Description). In IFCAR 2010 (LNCS), J. Giesl and R. Hähnle (Eds.). 15-21.

[20] Florian Rabe and Carsten Schürmann. 2009. A Practical Module System for LF. In Proceedings of the Fourth International Workshop on Logical Frameworks and Meta-Languages: Theory and Practice (LFMTP '09). ACM, New York, NY, USA, 40-48. https://doi.org/10.1145/1577824.1577831

[21] Alwen Tiu. 2004. A Logical Framework for Reasoning about Logical Specifications. Ph.D. Dissertation. Pennsylvania State University. http://etda.libraries.psu.edu/ theses/approved/WorldWideIndex/ETD-479/

[22] Yuting Wang. 2016. A Higher-Order Abstract Syntax Approach to the Verified Compilation of Functional Programs. Ph.D. Dissertation. University of Minnesota.

[23] Yuting Wang and Gopalan Nadathur. 2016. A Higher-Order Abstract Syntax Approach to Verified Transformations on Functional Programs. In ESOP 2016 (LNCS), Peter Thiemann (Ed.), Vol. 9632. Springer, 752-779. 